\title{
A New Spectral Bound on the Clique Number of Graphs
}

\author{
Samuel Rota Bulò and Marcello Pelillo \\ Dipartimento di Informatica - University of Venice - Italy \\ \{srotabul,pelillo\}@dsi.unive.it
}

\begin{abstract}
Many computer vision and patter recognition problems are intimately related to the maximum clique problem. Due to the intractability of this problem, besides the development of heuristics, a research direction consists in trying to find good bounds on the clique number of graphs. This paper introduces a new spectral upper bound on the clique number of graphs, which is obtained by exploiting an invariance of a continuous characterization of the clique number of graphs introduced by Motzkin and Straus. Experimental results on random graphs show the superiority of our bounds over the standard literature.
\end{abstract}

\section{Introduction}

Many problems in computer vision and pattern recognition can be formulated in terms of finding a completely connected subgraph (i.e. a clique) of a given graph, having largest cardinality. This is called the maximum clique problem (MCP). One popular approach to object recognition, for example, involves matching an input scene against a stored model, each being abstracted in terms of a relational structure 1234, and this problem, in turn, can be conveniently transformed into the equivalent problem of finding a maximum clique of the corresponding association graph. This idea was pioneered by Ambler et. al. [5] and was later developed by Bolles and Cain [6] as part of their local-feature-focus method. Now, it has become a standard technique in computer vision, and has been employing in such diverse applications as stereo correspondence [7, point pattern matching 8], image sequence analysis 9 . Other interesting applications of the maximum clique problem arise in the context of cluster analysis, where graph-theoretical methods have long proven to be especially effective [101112], and in the context of category learning and knowledge discovery [1314. Furthermore, clique finding is also linked with the learning of graphical structure by the Hammersley-Clifford theorem [15.

From a computational point of view, the maximum clique problem (MCP) belongs to the class of NP-Complete problems, whose intractability forces us to fall back on approximation methods. Unfortunately, even approximating the MCP is intractable [16]. Due to this pessimistic state of affairs, much attention has gone into developing efficient heuristics for the MCP, for which no formal guarantee of performance may be provided, but are nevertheless useful in practical 
applications. We refer to Bomze et al. [17] for a survey concerning algorithms, applications, and complexity issues of this important problem.

Another interesting direction of research consists in trying to bound the clique number of a graph. In the literature we find both upper and lower bounds [18]. The former however are in general more interesting because any heuristics for the MCP can be used to generate lower bounds. In this paper we propose a new spectral upper bound by exploiting an invariance of a continuous characterization of the clique number of graphs introduced by Motzkin and Straus [19], and we present an algoritm for efficiently computing the bound. Experiments on random graphs demonstrate the effectiveness of our result. The bound proposed here can be used in the bounding phase of branch-and-bound style algorithms for finding maximal cliques, with applications in such problems as graph matching [120] and clustering [12] (see also [21] for the use of bounds in graph matching problems).

\section{Bounds on the Clique Number of Graphs}

Let $G=(V, E)$ be a (undirected) graph, where $V=\{1, \ldots, n\}$ is the vertex set and $E \subseteq\left(\begin{array}{l}V \\ 2\end{array}\right)$ is the edge set, with $\left(\begin{array}{l}V \\ k\end{array}\right)$ denoting the set of all $k$-element subsets of $V$. A clique of $G$ is a subset of mutually adjacent vertices in $V$. A clique is called maximal if it is not contained in any other clique. A clique is called maximum if it has maximum cardinality. The maximum size of a clique in $G$ is called the clique number of $G$ and is denoted by $\omega(G)$.

Several spectral bounds on the clique number of graphs have been inspired by a theorem due to Motzkin and Straus 19. This result establishes a link between the problem of finding the clique number of a graph $G$ and the problem of optimizing the Lagrangian of $G$ over the simplex $\Delta$, where the Lagrangian of a graph $G=(V, E)$ is the function $L_{G}: \mathbb{R}^{n} \rightarrow \mathbb{R}$ defined as

$$
L_{G}(\mathbf{x})=\sum_{\{i, j\} \in E} x_{i} x_{j},
$$

and the standard simplex $\Delta$ is the set of nonnegative $n$-dimensional real vectors that sum up to 1 , i.e., $\Delta=\left\{\mathbf{x} \in \mathbb{R}_{+}^{n}: \sum_{i=1}^{n} x_{i}=1\right\}$.

Theorem 1 (Motzkin-Straus). Let $G$ be a graph with clique number $\omega(G)$, and $\mathbf{x}^{*}$ a maximizer of $L_{G}$ over $\Delta$ then

$$
L_{G}\left(\mathrm{x}^{*}\right)=\frac{1}{2}\left[1-\frac{1}{\omega(G)}\right] \text {. }
$$

Assuming $S$ a maximum clique of $G$, Motzkin and Straus additionally proved that the characteristic vector $\mathbf{x}^{S}$ of $S$ defined as

$$
x_{i}^{S}= \begin{cases}\frac{1}{|S|} & i \in S \\ 0 & i \notin S\end{cases}
$$

is a global maximizer of $L_{G}$ over $\Delta$. 
Before reviewing some existing bounds on $\omega$, we briefly introduce some concepts from spectral graph theory. The spectral radius $\rho(G)$ of a graph $G$ is the largest eigenvalue of the adjacency matrix of $G$. An eigenvector of unit length having $\rho(G)$ as eigenvalue will be called Perron eigenvector of $G$. The Perron eigenvector is always nonnegative and it may not be unique unless the multiplicity of the largest eigenvalue is exactly 1 . By definition, the spectral radius $\rho$ and an associated Perron eigenvector $\mathbf{x}_{P}$ of a graph $G$ satisfy the eigenvalue equation

$$
A_{G} \mathbf{x}_{P}=\rho \mathbf{x}_{P},
$$

which can be equivalently expressed in terms of the graph Lagrangian $L_{G}$ as follows

$$
\nabla L_{G}\left(\mathbf{x}_{P}\right)=\rho \mathbf{x}_{P},
$$

where $\nabla$ is the standard gradient operator. Since $G$ is undirected and hence, $A_{G}$ is symmetric, a useful variational characterization of $\rho$ and $\mathbf{x}_{P}$ is given by the following constrained program,

$$
\rho=\max _{\mathbf{x} \in S_{2}} \mathbf{x}^{T} A_{G} \mathbf{x}=2 \max _{\mathbf{x} \in S_{2}} L_{G}(\mathbf{x})
$$

where $S_{k}=\left\{\mathbf{x} \in \mathbb{R}^{n}:\|\mathbf{x}\|_{k}^{k}=1\right\}$. Note that the eigenvectors of $A_{G}$ are the critical points of this maximization problem. A further alternative characterization of the spectral radius and Perron eigenvector, that will be useful in the sequel, consists in maximizing the Rayleigh quotient, i.e.,

$$
\rho=\max _{\mathbf{x} \in \mathbb{R}^{n}} \frac{\mathbf{x}^{T} A_{G} \mathbf{x}}{\mathbf{x}^{T} \mathbf{x}}=2 \max _{\mathbf{x} \in \mathbb{R}^{n}} \frac{L_{G}(\mathbf{x})}{\mathbf{x}^{T} \mathbf{x}} .
$$

Note that every eigenvector associated to $\rho$ is a maximizer in (2), whereas in (11) only a Perron eigenvector is a global maximizer.

We present now two upper bounds for $\omega$ that turned out to be the tightest ones in a paper of Budinich [18, where different bounds have been compared on random graphs. For a review of further spectral bounds we refer to [1822].

The fist upper bound can be obtained by exploiting both the Motzkin-Straus theorem and (2).

Theorem 2. Let $G$ be an undirected graph with clique number $\omega(G)$ and spectral radius $\rho$. Then

$$
\omega(G) \leq \rho+1
$$

Proof. Let $\mathbf{x}_{\omega}$ be the characteristic vector of a maximum clique of $G$, then $\mathbf{x}_{\omega}^{T} \mathbf{x}_{\omega}=1 / \omega(G)$ and by the Motzkin-Straus theorem $\mathbf{x}_{\omega}^{T} A_{G} \mathbf{x}_{\omega}=1-1 / \omega(G)$. By (2) we have that

$$
\frac{\mathbf{x}_{\omega}^{T} A_{G} \mathbf{x}_{\omega}}{\mathbf{x}_{\omega}^{T} \mathbf{x}_{\omega}}=\frac{1-\frac{1}{\omega(G)}}{\frac{1}{\omega(G)}}=\omega(G)-1 \leq \rho,
$$

from which the property derives. 
This bound can also be derived as a straightforward implication of the result of Wilf [23. The second bound is due to Amin and Hakimi [24]:

Theorem 3. Let $G$ be an undirected graph with adjacency matrix $A_{G}$ and clique number $\omega(G)$. Moreover, let $N_{-1}$ be the number of eigenvalues of $A_{G}$ that are less or equal to -1 . Then

$$
\omega(G) \leq N_{-1}+1
$$

\section{The $\eta$-Bound}

We will introduce a new class of upper bounds generalizing (B1), where we exploit the fact that the maximizers of the Motzkin-Straus formulation are invariant with respect to shifts of the adjacency matrix of a graph $G$, whereas the maxima and the spectrum of the shifted matrix are not. Our intuition is that we can tighten (B1) by opportunely shifting the adjacency matrix of $G$.

We define

$$
\phi_{G}(t, \mathbf{x})=\mathbf{x}^{T}\left[A_{G}+(t-1) \mathbf{1 1} \mathbf{1}^{\top}\right] \mathbf{x},
$$

where $\mathbf{1}$ is an opportunely sized column vector of all 1's. Then by the MotzkinStraus theorem we have

$$
\max _{\mathbf{x} \in \Delta} \phi_{G}(t, \mathbf{x})=\mathbf{x}^{T}\left[A_{G}+(t-1) \mathbf{1} \mathbf{1}^{\top}\right] \mathbf{x}=t-\frac{1}{\omega(G)} .
$$

We will denote with $\phi_{G}(t)$ the leading eigenvalue of $A_{G}+(t-1) \mathbf{1 1}^{\top}$, i.e.,

$$
\phi_{G}(t)=\max _{\mathbf{x} \in S_{2}} \phi_{G}(t, \mathbf{x})
$$

and with $\Phi_{G}(t)$ the set of eigenvectors associated to $\phi_{G}(t)$, i.e.,

$$
\Phi_{G}(t)=\underset{\mathbf{x} \in S_{2}}{\arg \max } \phi_{G}(t, \mathbf{x}) .
$$

Theorem 4 (t-bound). Let $G$ be a graph with adjacency matrix $A_{G}$ and clique number $\omega(G)$. Then for any $t>0$

$$
\omega(G) \leq \frac{\phi_{G}(t)+1}{t}
$$

Proof. Let $\mathbf{x}_{\omega}$ be the characteristic vector of a maximum clique of $G$. Then

$$
\phi_{G}(t) \geq \phi_{G}\left(t, \frac{\mathbf{x}_{\omega}}{\left\|\mathbf{x}_{\omega}\right\|_{2}}\right)=\frac{\phi_{G}\left(t, \mathbf{x}_{\omega}\right)}{\mathbf{x}_{\omega}^{T} \mathbf{x}_{\omega}}=\frac{t-\frac{1}{\omega(G)}}{\frac{1}{\omega(G)}}=\omega(G) t-1,
$$

from which the result follows. 
Theorem 4 introduces a class of upper bounds that contains (B1) as the special case $t=1$. Let us define the $t$-bound as

$$
\eta_{G}(t)=\frac{\phi_{G}(t)+1}{t}
$$

Of course the more interesting $t$-bound is the tightest one, which will be called $\eta$-bound and denoted by $\eta(G)$, i.e.,

$$
\eta(G)=\inf _{t>0} \eta_{G}(t)
$$

Note that $\eta(G)$ is well defined, because by Theorem 4 it is lower bounded by the clique number of $G$.

\section{Computation of the $\eta$-Bound}

This section is dedicated to showing that the computation of $\eta(G)$ is not difficult, although not obvious at first glance, and we will provide an efficient algorithm for its computation.

Proposition 1. Let $s>t>0$. For any $\mathbf{x}(s) \in \Phi_{G}(s)$ and $\mathbf{x}(t) \in \Phi_{G}(t)$ we have

$$
(s-t) \mathbf{x}(t)^{T} \mathbf{1 1}^{\top} \mathbf{x}(t) \leq \phi_{G}(s)-\phi_{G}(t) \leq(s-t) \mathbf{x}(s)^{T} \mathbf{1 1}^{\top} \mathbf{x}(s) .
$$

Proof

$$
\begin{aligned}
\phi_{G}(s)-\phi_{G}(t) & =\phi_{G}(s, \mathbf{x}(s))-\phi_{G}(t) \\
& =\phi_{G}(t, \mathbf{x}(s))+(s-t) \mathbf{x}(s)^{T} \mathbf{1 1}^{\top} \mathbf{x}(s)-\phi_{G}(t) \\
& \leq \phi_{G}(t, \mathbf{x}(t))+(s-t) \mathbf{x}(s)^{T} \mathbf{1 1}^{\top} \mathbf{x}(s)-\phi_{G}(t) \\
& =\phi_{G}(t)+(s-t) \mathbf{x}(s)^{T} \mathbf{1 1}^{\top} \mathbf{x}(s)-\phi_{G}(t) \\
& =(s-t) \mathbf{x}(s)^{T} \mathbf{1 1}^{\top} \mathbf{x}(s) \\
\phi_{G}(s)-\phi_{G}(t) & =\phi_{G}(s, \mathbf{x}(s))-\phi_{G}(t) \\
& \geq \phi_{G}(s, \mathbf{x}(t))-\phi_{G}(t) \\
& =\phi_{G}(t, \mathbf{x}(t))+(s-t) \mathbf{x}(t)^{T} \mathbf{1} \mathbf{1}^{\top} \mathbf{x}(t)-\phi_{G}(t) \\
& =\phi_{G}(t)+(s-t) \mathbf{x}(t)^{T} \mathbf{1} \mathbf{1}^{\top} \mathbf{x}(t)-\phi_{G}(t) \\
& =(s-t) \mathbf{x}(t)^{T} \mathbf{1} \mathbf{1}^{\top} \mathbf{x}(t)
\end{aligned}
$$

Proposition 2. Let $s>t>0$. For any $\mathbf{x}(s) \in \Phi_{G}(s)$ and $\mathbf{x}(t) \in \Phi_{G}(t)$ the following propositions hold

1. if $\phi_{G}(0, \mathbf{x}(s)) \geq-1$ then $\eta_{G}(s) \leq \eta_{G}(t)$,

2. if $\phi_{G}(0, \mathbf{x}(t)) \leq-1$ then $\eta_{G}(s) \geq \eta_{G}(t)$. 
Proof. If $\phi_{G}(0, \mathbf{x}(s)) \geq-1$ then

$$
\begin{aligned}
\eta_{G}(s)-\eta_{G}(t) & =\frac{\phi_{G}(s)+1}{s}-\frac{\phi_{G}(t)+1}{t} \\
& \leq \frac{\phi_{G}(t)+(s-t) \mathbf{x}(s)^{T} \mathbf{1 1}^{\top} \mathbf{x}(s)+1}{s}-\frac{\phi_{G}(t)+1}{t} \quad \text { (by Prop. 1) } \\
& =\frac{(s-t)\left[-\phi_{G}(t)-1+t \mathbf{x}(s)^{T} \mathbf{1} \mathbf{1}^{\top} \mathbf{x}(s)\right]}{t s} \\
& \leq \frac{(s-t)\left\{-\phi_{G}(t, \mathbf{x}(s))-1+t \mathbf{x}(s)^{T} \mathbf{1} \mathbf{1}^{\top} \mathbf{x}(s)\right\}}{t s} \\
& =\frac{(s-t)\left[-\phi_{G}(0, \mathbf{x}(s))-1\right]}{t s} \leq 0 .
\end{aligned}
$$

While if $\phi_{G}(0, \mathbf{x}(t)) \leq-1$ then

$$
\begin{aligned}
\eta_{G}(s)-\eta_{G}(t) & =\frac{\phi_{G}(s)+1}{s}-\frac{\phi_{G}(t)+1}{t} \\
& \geq \frac{\phi_{G}(t)+(s-t) \mathbf{x}(t)^{T} \mathbf{1 1}^{\top} \mathbf{x}(t)+1}{s}-\frac{\phi_{G}(t)+1}{t} \quad \text { (by Prop. 1) } \\
& =\frac{(s-t)\left[-\phi_{G}(t)-1+t \mathbf{x}(t)^{T} \mathbf{1 1}^{\top} \mathbf{x}(t)\right]}{t s} \\
& =\frac{(s-t)\left[-\phi_{G}(0, \mathbf{x}(t))-1\right]}{t s} \geq 0 .
\end{aligned}
$$

Theorem 5. Let $s>t>0$. For any $\mathbf{x}(s) \in \Phi_{G}(s)$ and $\mathbf{x}(t) \in \Phi_{G}(t)$ if

$$
\phi_{G}(0, \mathbf{x}(s)) \leq-1 \leq \phi_{G}(0, \mathbf{x}(t)),
$$

then there exists $t \leq q \leq s$ such that $\eta(G)=\eta_{G}(q)$.

Moreover, if for any $q>0$ and $\mathbf{x}(q) \in \Phi_{G}(q)$ we have $\phi_{G}(0, \mathbf{x}(q))=-1$ then $\eta(G)=\eta_{G}(q)$.

Proof. By Proposition 2 it follows that

- for any $r<t$ we have $\eta(G) \leq \eta_{G}(t) \leq \eta_{G}(r)$;

- for any $r>s$ we have $\eta(G) \leq \eta_{G}(s) \leq \eta_{G}(r)$,

from which the first part of the result follows.

For the second part note that for any $r<q$ we have $\eta(G) \leq \eta_{G}(q) \leq \eta_{G}(r)$, while for any $r>q$ we have $\eta(G) \leq \eta_{G}(q) \leq \eta_{G}(r)$. Hence, $\eta_{G}(q)=\eta(G)$.

Proposition 3. Let $\mathbf{x}(1) \in \Phi_{G}(1)$ be a Perron eigenvector of $A_{G}$. For any $\mathbf{x}(0) \in \Phi_{G}(0)$ we have

$$
\phi_{G}(0, \mathbf{x}(1)) \leq-1 \leq \phi_{G}(0, \mathbf{x}(0)) .
$$




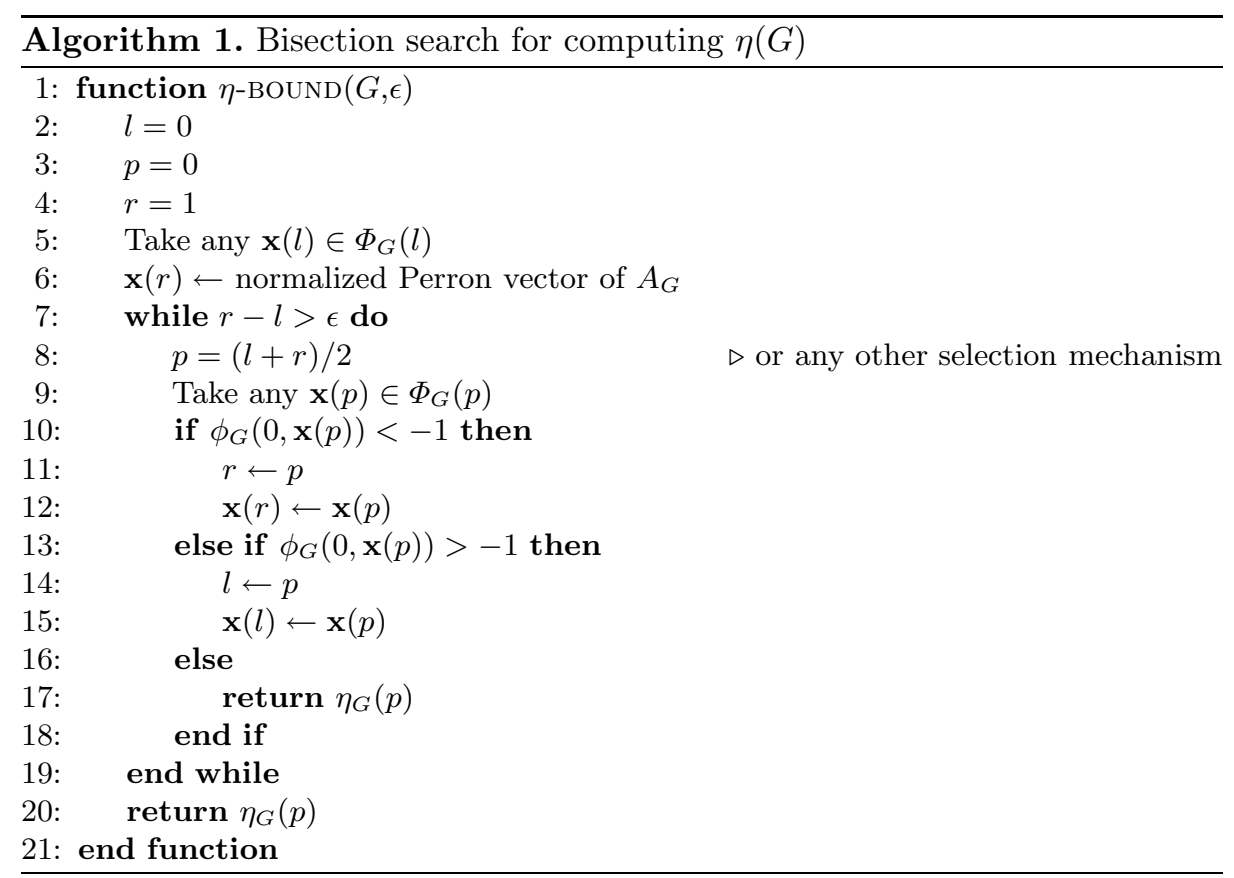

Proof. It follows from (3) that $\phi_{G}(0)=\phi_{G}(0, \mathbf{x}(0)) \geq-1$.

Because of the nonnegativity of the Perron vector, trivially $\mathbf{x}(1)^{T} A_{G} \mathbf{x}(1) \leq$ $\mathbf{x}(1)^{T}\left(\mathbf{1 1}^{\top}-I\right) \mathbf{x}(1)$, from which it follows that

$$
\begin{aligned}
& \phi_{G}(0, \mathbf{x}(1))+1= \\
& \quad=\mathbf{x}(1)^{T}\left[A_{G}-\mathbf{1 1}^{\top}\right] \mathbf{x}(1)+1 \leq \mathbf{x}(1)^{T}\left[\left(\mathbf{1 1}^{\top}-I\right)-\mathbf{1 1}^{\top}\right] \mathbf{x}(1)+1=0 .
\end{aligned}
$$

Theorem [5] and Proposition 3 suggest an effective way of computing $\eta(G)$ by performing a section search (like the bisection search) in the interval $(0,1]$. Indeed, Theorem 5 allows us to bisect an interval having sign-discording values of $f(t)=\phi_{G}(0, \mathbf{x}(t))+1$ at the endpoints, and restrict the attention to the subinterval that preserves this property. Proposition 3 , instead, entitles us to start the search procedure from the interval $[0,1]$. Note that we can stop the search if we encounter an endpoint $t$, where $f(t)=0$, as in this case $\eta_{G}(t)$ is our $\eta$-bound. Otherwise, the size of the interval is an indicator of the precision of the solution and we can stop as soon as this is small enough. Algorithm 1 reports an implementation that can be used for the computation of $\eta(G)$ with an arbitrary precision $\epsilon$.

\section{Experiments on Random Graphs}

In this section, we evaluate the performance of our $\eta$-bound. We compare our bound against other spectral bounds, which were the best performing approaches 
Table 1. Experiments on random graphs. The columns $n, \delta$ and $\omega$ are the order, density and average clique number of the random graphs, respectively. The results, expecting the last row, are expressed in terms of relative error.

\begin{tabular}{|c|c|c||r|r|r|}
\hline \multicolumn{2}{|c||}{ Random graphs } & \multicolumn{3}{c|}{ Bound errors } \\
\hline \multicolumn{1}{|c|}{$\delta$} & \multicolumn{1}{c|}{$\omega$} & $(\bar{B} 1)$ & $($ B2 $)$ & \multicolumn{1}{c|}{$\eta$} \\
\hline 100 & 0.05 & 3.12 & 1.25 & 10.58 & $\mathbf{0 . 7 9}$ \\
& 0.10 & 3.96 & 1.99 & 9.26 & $\mathbf{0 . 8 7}$ \\
& 0.20 & 5.00 & 3.33 & 7.84 & $\mathbf{1 . 0 7}$ \\
& 0.30 & 6.13 & 4.17 & 6.52 & $\mathbf{1 . 1 1}$ \\
& 0.40 & 7.51 & 4.49 & 5.24 & $\mathbf{1 . 0 8}$ \\
0.50 & 9.11 & 4.58 & 4.19 & $\mathbf{1 . 0 2}$ \\
0.60 & 11.51 & 4.28 & 3.16 & $\mathbf{0 . 9 1}$ \\
0.70 & 14.55 & 3.85 & 2.33 & $\mathbf{0 . 8 4}$ \\
& 0.80 & 19.99 & 3.03 & 1.45 & $\mathbf{0 . 6 4}$ \\
& 0.90 & 30.69 & 1.94 & 0.61 & $\mathbf{0 . 4 2}$ \\
& 0.95 & 43.50 & 1.19 & $\mathbf{0 . 1 6}$ & 0.27 \\
\hline 200 & 0.10 & 4.17 & 4.25 & 19.97 & $\mathbf{1 . 4 5}$ \\
& 0.50 & 11.00 & 8.19 & 7.71 & $\mathbf{1 . 4 7}$ \\
& 0.90 & $?$ & 180.10 & 99.08 & $\mathbf{6 8 . 4 5}$ \\
\hline
\end{tabular}

reviewed in the work of Budinich [18. Specifically, we compare against bounds (B1) and (B2), which have been previously introduced.

Table 1 reports the results obtained on random graphs, where $\eta$ is the column relative to our $\eta$-bound. The columns $n, \delta$ and $\omega$ are the order, density and average clique number of the random graphs, respectively. The results, except the last row, are expressed in terms of relative error, i.e. if $\bar{\omega}$ is the value of the bound then the relative error for the upper and lower bounds are $(\bar{\omega}-\omega) / \omega$ and $(\omega-\bar{\omega}) / \omega$, respectively. In the last row, where the average clique number could not be computed, we reported the absolute value of the bounds. It is clear that, as expected, our $\eta$-bound improves (B1). Moreover, our bound outperforms also Amin's one on all instances excepting very dense graphs. Interestingly, it exhibits on average a remarkable improvement over the competitors by keeping an overall small relative error.

\section{Conclusions}

In this paper, we introduced a new spectral bounds on the clique number of graphs, called $\eta$-bound, which has been obtained by combining spectral graph theory with a result due to Motzkin and Straus. Specifically, we exploit an invariance of the Motzkin-Sraus formulation with respect to shifts of the adjacency matrix of graphs in order to tighten a well-known bound. 
Finally, we tested our bounds on random graphs comparing them against state-of-the-art spectral approaches. The results outlined a marked improvement over the competitors.

\section{References}

1. Barrow, H., Burstall, R.M.: Subgraph isomorphism, matching relational structures and maximal cliques. Information Processing Letters 4(4), 83-84 (1976)

2. Chin, R.T., Dyer, C.R.: Model-based recognition in robot vision. Comput. Surveys 18(1), 67-108 (1986)

3. Pelillo, M., Siddiqi, K., Zucker, S.W.: Matching hierarchical structures using association graphs. IEEE Trans. Pattern Anal. Machine Intell. 21(11), 1105-1120 (1999)

4. Suetens, P., Fua, P., Hanson, A.J.: Computational strategies for object recognition. Comput. Surveys 24(1), 5-61 (1992)

5. Ambler, A.P., Barrow, H.G., Brown, C.M., Burstall, R.M., Popplestone, R.J.: A versatile computer-controlled assembly. In: Int. Joint Conf. on Artif. Intell., pp. 298-307 (1973)

6. Bolles, R.C., Cain, R.A.: Recognizing and locating partially visible objects: the local-feature-focus method. Int. J. Robotics Res. 1(n), 57-82 (1982)

7. Horaud, R., Skordas, T.: Stereo correspondence through feature grouping and maximal cliques. IEEE Trans. Pattern Anal. Machine Intell. 11(11), 1168-1180 (1989)

8. Ogawa, H.: Labeled point pattern matching by delaunay triangulation and maximal cliques. Pattern Recogn. 19(1), 35-40 (1986)

9. Radig, B.: Image sequence analysis using relational structures. Pattern Recogn. 17(1), 161-167 (1984)

10. Augustson, J.G., Minker, J.: An analysis of some graph theoretical cluster techniques. J. ACM 17(4), 571-588 (1970)

11. Jain, A.K., Dubes, R.C.: Algorithms for data clustering. Prentice-Hall, Englewood Cliffs (1988)

12. Pavan, M., Pelillo, M.: Dominant sets and pairwise clustering. IEEE Trans. Pattern Anal. Machine Intell. 29(1), 167-172 (2007)

13. Dmitry, D., Ari, R.: Efficient unsupervised discovery of word categories using symmetric patterns and high frequency words. In: 21st Int. Conf. on Computational Linguistics and 44th Annual Meeting of the ACL, Association for Computational Linguistics, pp. 297-304 (2006)

14. Nina, M., Dana, R., Ram, S.: A new conceptual clustering framework. Machine Learning 56, 115-151 (2004)

15. Hammersley, J., Clifford, P.: Markov fields on finite graphs and lattices (1971)

16. Hastad, J.: Clique is hard to approximate within $n^{1-\varepsilon}$. In: Ann. Symp. Found. Comput. Sci., vol. 37, pp. 627-636 (1996)

17. Bomze, I.M., Budinich, M., Pardalos, P.M., Pelillo, M.: The maximum clique problem. In: Handbook of Combinatorial Optimization, vol. 1, pp. 1-74. Kluwer Academic Publishers, Boston (1999)

18. Budinich, M.: Exact bounds on the order of the maximum clique of a graph. Discr. Appl. Math. 127, 535-543 (2003) 
19. Motzkin, T.S., Straus, E.G.: Maxima for graphs and a new proof of a theorem of Turán. Canad. J. Math. 17, 533-540 (1965)

20. Pelillo, M.: Replicator equations, maximal cliques, and graph isomorphism. Neural Computation 11(8), 1933-1955 (1999)

21. Schellewald, C.: A bound for non-subgraph isomorphism. In: Escolano, F., Vento, M. (eds.) GbRPR. LNCS, vol. 4538, pp. 71-80. Springer, Heidelberg (2007)

22. Lu, M., Liu, H., Tian, F.: Laplacian spectral bounds for clique and independence numbers of graphs. J. Combin. Theory Series B 97(5), 726-732 (2007)

23. Wilf, H.S.: The eigenvalues of a graph and its chromatic number. J. London Math. Soc. 42, 330-332 (1967)

24. Amin, A.T., Hakimi, S.L.: Upper bounds of the order of a clique of a graph. SIAM J. on Appl. Math. 22(4), 569-573 (1972) 